\title{
Attosecond time-delay spectroscopy of the hydrogen molecule
}

\author{
I. A. Ivanov* and A. S. Kheifets \\ Research School of Physical Sciences, The Australian National University, Canberra ACT 0200, Australia \\ Vladislav V. Serov \\ Department of Theoretical Physics, Saratov State University, 83 Astrakhanskaya, Saratov 410012, Russia
}

(Received 24 July 2012; published 27 December 2012)

\begin{abstract}
We apply the concept of photoemission time delay to the process of single-photon one-electron ionization of the $\mathrm{H}_{2}$ molecule. We demonstrate that, by resolving the photoelectron detection in time on the attosecond scale, one can extract differential photoionization cross sections for particular field and molecule orientations from the measurement on a randomly oriented molecule
\end{abstract}

DOI: 10.1103/PhysRevA.86.063422

PACS number(s): $32.80 . \mathrm{Rm}, 32.80 . \mathrm{Fb}, 42.50 . \mathrm{Hz}$

\section{INTRODUCTION}

Attosecond science has extended experimental studies of atomic and molecular photoionization into a new dimension. In atoms, a time delay between absorption of an attosecond XUV pulse and subsequent emission of a photoelectron has been measured [1-3]. In molecules, electron localization and attosecond control have been demonstrated in pump-probe photoionization experiments [4,5]. Experimental time-delay studies in molecules are yet to be performed either by using the attosecond streaking [1] or by the interferometric sideband oscillation technique [2]. In the meantime, molecules offer a very rich and complex photoionization picture in which the cross section and angular distribution of photoelectrons depend sensitively on the molecular orientation relative to the polarization axis of VUV radiation. Thus the attosecond time delay studies can be very beneficial to molecular photoionization by defining the phase of the ionization amplitude and thus achieving the complete photoionization experiment [6].

In the simplest case of a homonuclear diatomic molecule, its orientation is defined by the mutual angle $\theta_{N}$ of the molecular and polarization axes. Photoionization cross section and angular distribution of photoelectrons in $\mathrm{H}_{2}$ and $\mathrm{D}_{2}$ depend strongly on this angle varying between the limits of $\Sigma\left(\theta_{N}=0\right)$ and $\Pi\left(\theta_{N}=90^{\circ}\right)$ orientations. In single photoionization, the amplitude and cross section of the $\Sigma$ orientated $\mathrm{H}_{2}$ display a deep minimum [7] which can be attributed to the two-center electron interference [8]. In double photoionization (DPI), the angular correlation pattern in two-electron continuum shows strong variation with the angle $\theta_{N}$. Because of the Coulomb explosion of the doubly ionized $\mathrm{H}_{2}$ molecule, its orientation at the moment of ionization can be measured experimentally $[9,10]$. In principle, neutral polarizable molecules can also be aligned by a strong laser field [11]. However, reports of single photoionization of aligned molecules are not known to the authors.

In the present paper, we offer an alternative strategy of attosecond studies of randomly oriented molecules. Within the framework of the time delay theory $[12,13]$ and by employing the saddle-point method, we figure out that the phase of the electron wave packet, emanated from the randomly oriented

\footnotetext{
*Igor.Ivanov@anu.edu.au
}

molecule, carries information about the angle differential photoionization cross section specific to certain molecular orientations. By extracting this information, one can effectively measure the orientation specific cross sections without actually aligning the molecule. In the following, we illustrate our findings by using a process of single-photon one-electron ionization of the $\mathrm{H}_{2}$ molecule as a convenient example.

\section{THEORY AND RESULTS}

We shall be interested in the probability density distribution $P(t)$ to detect an electron at the moment of time $t$ using a detector placed at a point $\boldsymbol{r}$ far away from the ionized molecule. The time-dependent wave function of the ejected electron after the end of the laser pulse can be written as

$$
\Psi(\boldsymbol{r}, t)=\int d \boldsymbol{q} f(\boldsymbol{q}) \Psi_{\boldsymbol{q}}^{-}(\boldsymbol{r}) e^{-i E t},
$$

where $\Psi_{q}^{-}(\boldsymbol{r})$ are the (ingoing) scattering states in the field of the molecular ion and $f(\boldsymbol{q})$ is the photoionization amplitude. A complete expansion of the wave function should include bound states as well but they do not propagate to large distances and hence do not affect the asymptotic behavior of the wave packet, so we omit them. For large $t$ and $r$, the integral in Eq. (1) can be evaluated using the saddle-point method. For the wave packet, describing an electron escaping with the asymptotic momentum $\boldsymbol{k}$, the amplitude $|f(\boldsymbol{q})|$ in Eq. (1) can be represented near its maximum as $f(\boldsymbol{q}) \simeq \exp \left[-a\left(q_{\|}-\right.\right.$ $\left.k)^{2}+i \delta(\boldsymbol{q})-b q_{\perp}^{2}\right]$, where $\boldsymbol{q}_{\|}$and $\boldsymbol{q}_{\perp}$ are the components of the vector $\boldsymbol{q}$ in the direction of asymptotic momentum $\boldsymbol{k}$ and perpendicular direction, respectively. The quantity $\delta(\boldsymbol{q})$ is the phase of the ionization amplitude. The parameter $b$ characterizes the spread of the wave packet in the lateral direction, and it is determined ultimately by the experimental geometry. The parameter $a$ characterizes the spread of the wave packet in the direction of the momentum vector $\boldsymbol{k}$ towards the detector. If the wave packet is well collimated and the parameter $b$ is large, so that $b \gg a$, then the energy spread of the wave packet is approximately $\Delta E \approx k / \sqrt{a}$ which, in turn, is equal approximately to the bandwidth of the driving laser pulse.

The large $t$ asymptotic behavior of the wave function (1) is determined by the $q_{\|}$integration. The scattering states in Eq. (1) are asymptotically Coulomb waves $\Psi_{\boldsymbol{q}}^{-} \propto e^{i \boldsymbol{q} \cdot \boldsymbol{r}+i \gamma(\boldsymbol{r}, \boldsymbol{q})}$ 
with $\gamma(\boldsymbol{r}, \boldsymbol{q})=q^{-1} \ln (r q+\boldsymbol{r} \cdot \boldsymbol{q}) \quad[1,14,15]$. Therefore, the saddle point, that determines the large $t$ behavior of the integral in Eq. (1), is a critical point of the expression

$$
\begin{aligned}
S\left(\boldsymbol{q}_{\|}, t\right)= & -a\left(q_{\|}-k\right)^{2}+i \delta\left(q_{\|}\right)-i q_{\|}^{2} t / 2+i \boldsymbol{q}_{\|} \boldsymbol{r} \\
& +i \gamma\left(\boldsymbol{r}, \boldsymbol{q}_{\|}\right) .
\end{aligned}
$$

This critical point determines the asymptotic electron trajectory

$$
\boldsymbol{r}(t) \asymp \boldsymbol{k}\left(t-t_{0}\right)+\boldsymbol{r}^{\prime}(t) .
$$

Here $t_{0}(E)=k^{-1} d \delta / d q_{\|}$is the time delay and $\boldsymbol{r}^{\prime}(t)=$ $-d \gamma\left(\boldsymbol{r}, \boldsymbol{q}_{\|}\right) / d \boldsymbol{q}_{\|}$is a known function varying logarithmically slow with $t$. All the derivatives here are assumed to be taken at the point $\boldsymbol{q}_{\|}=\boldsymbol{k}$.

To find the probability of the electron detection as a function of time, we have to evaluate the integral in Eq. (1). By using the saddle-point method, expanding Eq. (2) around the critical point, and retaining the quadratic terms, we arrive at the following expression:

$$
\Psi(\boldsymbol{r}, t) \propto \exp \left\{-\frac{\left[\boldsymbol{r}-\boldsymbol{k}\left(t-t_{0}\right)-\boldsymbol{r}^{\prime}(t)\right]^{2}}{4 a}\right\} .
$$

The squared modulus of the wave function (4) defines the probability $P\left(t, \boldsymbol{R}, \theta_{N}\right)$ for an electron to arrive at the moment of time $t$ at the detector placed at the point $\boldsymbol{R}=\boldsymbol{n} R$ :

$$
P\left(t, \boldsymbol{R}, \theta_{N}\right)=A\left(\boldsymbol{n}, \theta_{N}\right) \exp \left\{-\frac{k^{2}\left[t-t_{0}\left(\boldsymbol{n}, \theta_{N}\right)-\tau\right]^{2}}{2 a}\right\} .
$$

Here we introduced the arrival time $\tau$ defined as a root of the equation:

$$
\boldsymbol{R}=\boldsymbol{k} \tau+\boldsymbol{r}^{\prime}(\tau)
$$

From Eq. (6) and the definition of the function $\boldsymbol{r}^{\prime}(t)$ above, it is clear that the arrival time depends only on the experimental geometry and does not depend on the field and molecule orientation. The physical meaning of the arrival time is obvious. It corresponds to the moment of time at which the distribution of the electrons, arriving at the detector, as a function of time would have peaked in the absence of any time delay. When deriving Eq. (5), we also took account of the fact that both the preexponential factor and the time delay $t_{0}$ depend on the unit vector $\boldsymbol{n}=\boldsymbol{R} / R$ in the direction of the detector and the mutual field and molecule orientation, defined by the angle $\theta_{N}$. The preexponential factor can be found by noting that the integration of $P\left(t, \boldsymbol{R}, \theta_{N}\right)$ over time should give us the total probability to detect an electron escaping in a given direction. The latter can be expressed in terms of the differential cross section and the total energy carried by the laser pulse [16]. For the coefficient in Eq. (5) we thus obtain

$$
A\left(\boldsymbol{n}, \theta_{N}\right)=\frac{k_{0} c}{8 \pi^{\frac{3}{2}} \omega} \frac{d \sigma\left(\boldsymbol{n}, \theta_{N}\right)}{d \Omega} \int F^{2}(t) d t .
$$

Here $c \approx 137$ is the speed of light in atomic units. The integral of the squared electric field intensity $F(t)$ is taken over the duration of the pulse. This integral is related to the total energy of the laser pulse which is usually known in the experiment. The signal measured at the detector is an average over all possible molecular orientations:

$$
P_{\text {avg }}(t)=\int_{0}^{\frac{\pi}{2}} P\left(t, \theta_{N}\right) \sin \theta_{N} d \theta_{N} .
$$

As a numerical example, we consider here the process of single-photon one-electron ionization of $\mathrm{H}_{2}$ for the photon energy $\omega=21.5 \mathrm{eV}$. We consider the geometry in which photoelectrons are detected in the $z$ direction of the polarization vector of the laser field. Numerical data, that are required to evaluate Eqs. (5) and (8), are computed using the exterior complex scaling method in prolate spheroidal coordinates (PSECS) [17]. These data are the angle differential cross section in the direction of the photoelectron detection and the time delay corresponding to the given photoelectron energy $E$. The latter is computed as

$$
t_{0}\left(\boldsymbol{n}, \theta_{N}\right)=\operatorname{Im}\left[\frac{d f\left(\boldsymbol{n}, \theta_{N}\right)}{d E} \frac{1}{f\left(\boldsymbol{n}, \theta_{N}\right)}\right],
$$

where $f\left(\boldsymbol{n}, \theta_{N}\right)$ is the photoionization amplitude. The energy derivative is evaluated using the finite difference formula by running PSECS calculations for two closely spaced energies. For the parameter $a$ in Eq. (5) we used the value $a=20$ a.u. For the carrier pulse frequency of $21.5 \mathrm{eV}$ (an example we consider below) this gives us the characteristic spread of the electron wave packet of $3 \mathrm{eV}$. We emphasize that the exact shape of the pulse is not relevant to our analysis, as long as the electric field of the pulse can be represented as $E(t)=$ $f(t) \cos \omega t$ (which is the form we employed in the calculation), or $E(t)=f(t) \sin \omega t$, where pulse envelope $f(t)$ is an even function on the interval $\left(-T_{1}, T_{1}\right)$ of the pulse duration. The phase of the amplitude in this case is not sensitive to the laser pulse shape as can be readily seen from the perturbation theory expression for the ionization amplitude. Therefore, as long as we consider the pulses of not very high intensity and shape described above, all we need for the saddle point derivation is a quadratic expansion of the modulus of the ionization amplitude near the critical point, which is always valid. In case of the pulse shape different from the shapes described above (which is the case of the chirped pulse, for example), we have to introduce an additional energy dependent phase factor in the expression for the amplitude. This will lead to replacement of the phase $\delta\left(q_{\|}\right)$in Eq. (2) with modified phase $\delta\left(q_{\|}\right)+\Delta\left(q_{\|}\right)$. Important point to note here is that $\Delta\left(q_{\|}\right)$does not depend on the molecular orientation. In the expression for the electron trajectory (3), introduction of this factor will, therefore, result only in the replacement of the time delay $t_{0}$ with modified delay $t_{0}+T$, where $T=k^{-1} d \Delta / d q_{\|}$, and is independent of the molecular orientation. Net effect of the introduction of the additional phase shift $\Delta\left(q_{\|}\right)$, is, thus, equivalent to shift of the time zero in all the subsequent equations.

Our numerical results are presented on the Fig. 1 in the form of the photoelectron detection probability distribution $P_{\text {avg }}(t)$ as a function of time measured from the moment of arrival $\tau$. On the same figure, we also display the probability distribution $P_{\text {avg }}^{0}(t)$, obtained if in Eq. (5) we put time delays to zero. The difference between the time delayed and zero delayed probabilities is highlighted on the right panel of Fig. 1 where we display the normalized difference signal $\left[P_{\mathrm{avg}}(t)-P_{\mathrm{avg}}^{0}(t)\right] / P_{\mathrm{avg}}^{0}(t)$. As can be seen from Eq. (5) and 

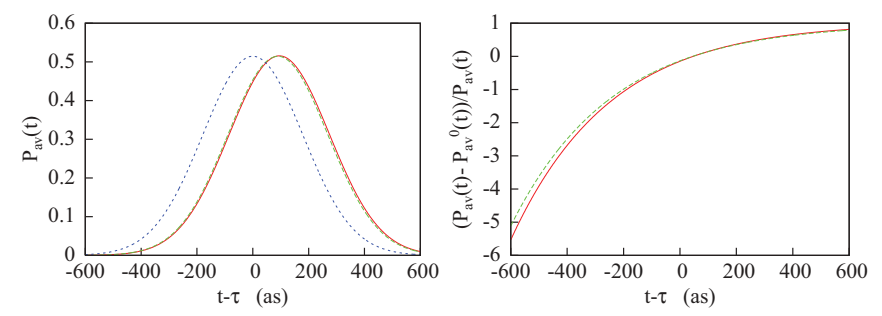

FIG. 1. (Color online) Left panel: angular averaged probability distribution $P_{\text {avg }}(t)$ computed according to Eqs. (5) and (8) is shown by the solid (red) line. The dash (green) line: results of the fitting procedure. The (blue) dots: distribution $P_{\mathrm{avg}}^{0}(t)$ computed assuming zero time delays in Eq. (5). Right panel: normalized difference signal $\left[P_{\mathrm{avg}}(t)-P_{\mathrm{avg}}^{0}(t)\right] / P_{\mathrm{avg}}^{0}(t)$. Photon energy $\omega=21.5 \mathrm{eV}$.

Fig. 1, the probability $P_{\mathrm{avg}}^{0}(t)$ is an even function of $t=\tau$ peaked at $t-\tau=0$. The time delays $t_{0}\left(\boldsymbol{n}, \theta_{N}\right)$, which are different for different orientations $\theta_{N}$, are responsible for the asymmetry of the averaged distribution visible on both panels of Fig. 1.

The computational procedure, that we described above, solves the direct problem of evaluating the probability distribution of the counts on the detector as a function of time. Now we demonstrate that the inverse problem can also be solved, i.e., one can extract information about the cross sections and time delays for particular values of field and molecule orientation using the averaged signal $P_{\text {avg }}(t)$. The amplitude $f\left(\theta_{N}\right)$ and its energy derivative can be parametrized as functions of the angle $\theta_{N}[18]$

$$
\begin{aligned}
f\left(\theta_{N}\right) & =\beta_{1} \cos \theta_{N}+\beta_{2} \sin \theta_{N}, \\
\frac{d f\left(\theta_{N}\right)}{d E} & =\beta_{3} \cos \theta_{N}+\beta_{4} \sin \theta_{N},
\end{aligned}
$$

where $\beta_{i}$ are some complex parameters. With these parameters, we can find the differential cross section and the time delay entering Eqs. (5) and (7) [for the time delay we use Eq. (9)]. The parameter $a$, which describes the momentum distribution of the electrons near the crest of the wave packet, is rarely, if ever, known in the experiment. We treat it, therefore, as an additional fitting parameter. This gives us a set of fitting parameters $\boldsymbol{\beta}, a$. With this set of parameters, we compute the trial distribution $P_{\text {trial }}(t)$ using Eqs. (5), (8), and (10). With $P_{\text {trial }}(t)$ thus computed, and $P_{\text {avg }}(t)$ presumed to be known, we form a functional

$$
d(\boldsymbol{\beta}, a)=\int_{-\infty}^{\infty}\left[P_{\text {trial }}(t)-P_{\mathrm{avg}}(t)\right]^{2} d t .
$$

By minimizing $d(\boldsymbol{\beta}, a)$ with respect to the fitting parameters, we find the amplitude as a function of the angle $\theta_{N}$. The result of such a fit for the probability distribution is shown in Fig. 1 for the photon energy $\omega=21.5 \mathrm{eV}$. The fitted curve is almost indistinguishable from the original calculation.

By knowing the optimal set of fitting parameters $\beta$, we can compute the cross section and the time delay as functions of the angle $\theta_{N}$. These results are shown in Fig. 2 for three fixed photon energies $\omega=21.5,64$, and $79 \mathrm{eV}$ along with the original data computed by the PSECS method. One can see that our fitting procedure reproduces the PSECS cross-section data quite reasonably except for the angles
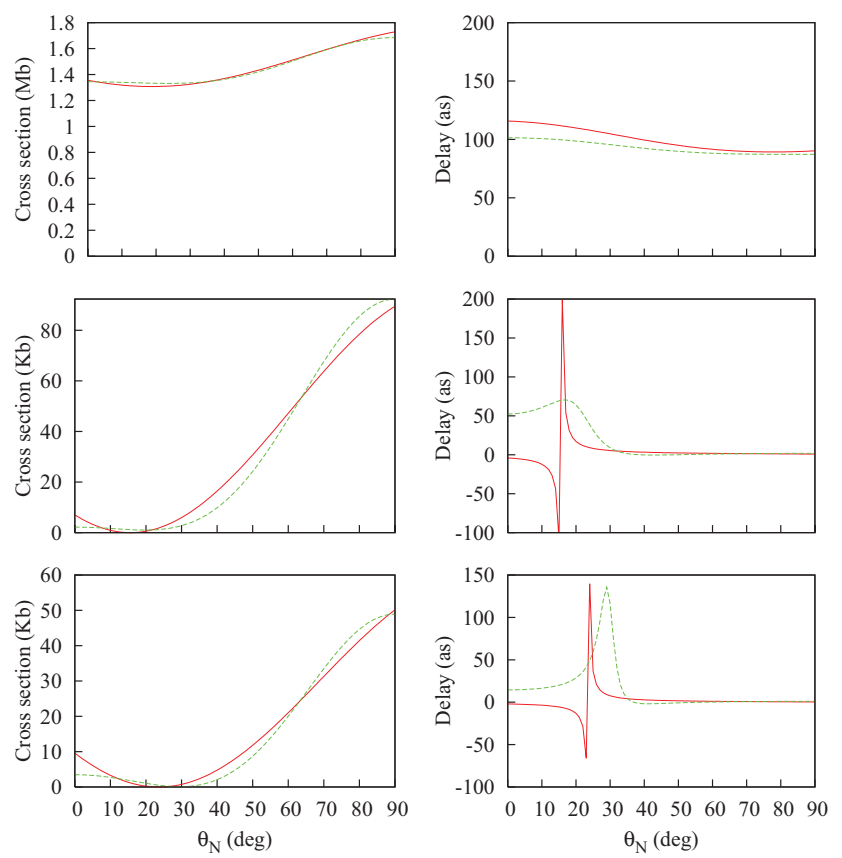

FIG. 2. (Color online) Differential cross sections of detecting the photoelectron in the $z$ direction (left column) and corresponding time delays (right column) as functions of angle between molecular axis and field direction for photon energies of $21.5 \mathrm{eV}$ (first row), $64 \mathrm{eV}$ (second row), and $79 \mathrm{eV}$ (lower row). Solid (red) line: data obtained using the fitting procedure; dash (green): calculated results.

$\theta_{N}$, where this cross section becomes small. That could be expected as the preexponential factor in Eq. (5) dampens heavily the probability making the fitting procedure insensitive to the contribution from these angles. Since the time delay is generally large when the cross section is small, the agreement of the fitted and exact time delays in Fig. 2 is not nearly as good as for the cross sections.

The interval of the photon energies that we considered includes the deep minimum at approximately $75 \mathrm{eV}$ for the $\Sigma$ orientation. The ratio of the cross sections $\sigma_{\Pi} / \sigma_{\Sigma}$ shows a prominent maximum at this photon energy [7], where it is approximately an order of magnitude larger than for the photon energies far from the minimum. This means that, for the photon energies near $75 \mathrm{eV}$, the cross section as a function of the angle $\theta_{N}$ varies much more on the interval $\theta_{N} \in(0, \pi / 2)$ than for the photon energies away from the minimum. Reproducing correctly a widely varying function using a fitting procedure is a more difficult task than reproducing a function which is nearly constant. We have, therefore, tested our procedure for the most challenging interval of the photon energies.

For practical implementations the technique we described must be robust in the sense that inaccuracies in the measured data, unavoidable in the experiment, do not alter significantly the results our procedure yields for the cross sections. In Fig. 3 we show results our procedure gives for the case when we introduce a random perturbation to the signal $P_{\text {avg }}(t)$ (we may call it the "experimental" signal). We also shifted the experimental signal along the time axis by the amount of $T=200$ as to model the uncertainty in the time zero moment which is hard to avoid in the experiment. To account for this overall shift along the time axis we added an additional fitting 

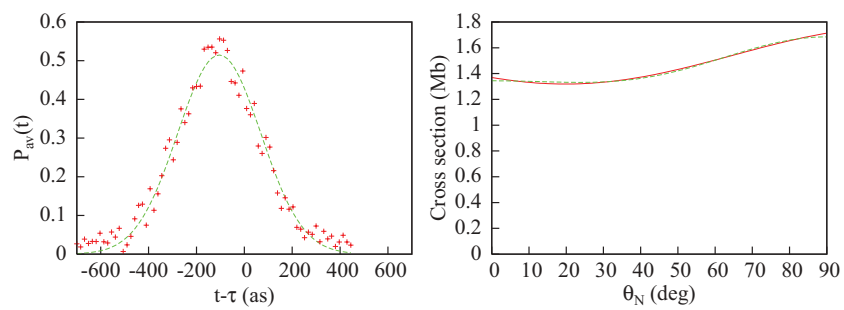

FIG. 3. (Color online) Left panel: crosses (red), experimental distribution $P_{\text {avg }}(t)$; dash (green) line, results of the fitting procedure. Right panel: differential cross section of detecting the photoelectron in the $z$ direction as function of the angle between molecular axis and field direction for photon energy of $21.5 \mathrm{eV}$.

parameter to the set of fitting parameters $\boldsymbol{\beta}, a$. As we noted above, the same effect [overall shift of the distribution $P_{\text {avg }}(t)$ along the time axis] can be produced by the pulse with carrier form different from sine or cosine forms.

Fitting procedure using the experimental signal as an input produces quite accurate results for the cross section.

Implementation of the above described procedure necessitates measuring development of the experimental signal in time with the resolution of the order of a few tens of attoseconds. To achieve that in practice one can employ the attosecond streaking technique by using the ionizing XUV pump and a streaking infrared (IR) probe [19,20]. This technique converts short intervals of time of the order of several attoseconds into more easily measurable physical characteristics such as photoelectron spectra. In the context of the present work this technique could be implemented using the procedure described in [21], or in our earlier work [22]. Let us apply to the system the IR pulse polarized in the $z$ direction, and arriving with delay $\Delta$ with respect to the driving laser pulse. Clearly, the IR field will modify the velocity distribution of the electrons arriving at the detector. We shall be interested in the distribution $P_{\text {avg }}\left(v_{z}\right)$ of the $z$ component of electron velocity. In the absence of the IR field, this distribution is an average over all molecular orientations analogous to the one in Eq. (8):

$$
P_{\mathrm{avg}}\left(v_{z}\right)=\int_{0}^{\frac{\pi}{2}}\left|f\left(q_{z}, \theta_{N}\right)\right|^{2} d \theta_{N} .
$$

Presence of the IR field modifies this distribution. Quantitatively, this effect can be described using the so-called softphoton approximation [23] as follows. Using the CoulombVolkov (CVA) approximation to describe the effect of the IR field on the molecule, one can obtain [21-23] the relation

$$
f^{1}\left(q_{z}, \theta_{N}\right)=\sum_{-\infty}^{\infty} f\left(q_{z m}, \theta_{N}\right)(-1)^{m} e^{-i m \Omega \Delta} J_{m}\left(\frac{k E_{\mathrm{IR}}^{0}}{\Omega^{2}}\right),
$$

where $f\left(q_{z}, \theta_{N}\right)$ is the amplitude of photoionization in $z$ direction for a particular field and molecule orientation in the presence of XUV pulse only, $f^{1}\left(q_{z}, \theta_{N}\right)$ is the amplitude in the presence of both XUV and IR pulses, and $k$ is the on-shell electron momentum. In Eq. (13) $J_{m}(x)$ is the Bessel function, $q_{z m}$ is defined so as to satisfy $q_{z m}^{2}=q_{z}^{2}-2 m \Omega$, and $E_{\mathrm{IR}}^{0}$ and $\Omega$ are amplitude and frequency of the IR field. We shall assume IR field intensity to be $3.5 \times 10^{10} \mathrm{~W} / \mathrm{cm}^{2}, \Omega_{\mathrm{IR}}=1 \mathrm{eV}$. Terms with different $m$ in Eq. (13) describe absorption or emission of

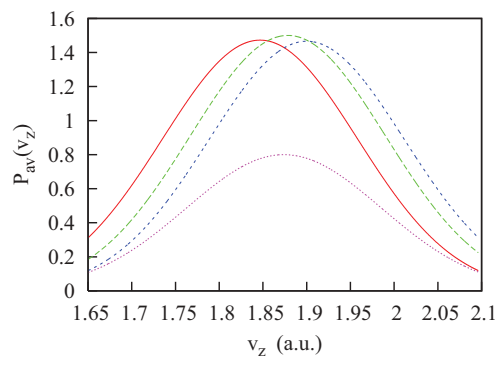

FIG. 4. (Color online) Distribution $P_{\text {avg }}\left(v_{z}\right)$ in the presence of IR field with intensity $3.5 \times 10^{10} \mathrm{~W} / \mathrm{cm}^{2}$ and frequency $\Omega_{\mathrm{IR}}=1 \mathrm{eV}$ for different values of the delay $\Delta$ between UV and IR pulses. Solid (red) line: $\Delta=0$; long dash (green): $\Delta=0.25 T_{\mathrm{IR}}$; dash (blue) $\Delta=0.5 T_{\mathrm{IR}}$; dots (magenta): velocity distribution without IR pulse scaled by a factor 0.5 for better visibility. $T_{\mathrm{IR}}$ is an optical cycle of the IR field. XUV photon energy $\omega=64 \mathrm{eV}$.

$m$ IR photons (only terms with $m=0, \pm 1$ need to be retained in this equation for the weak IR field we consider).

An alternative way to describe the effect of the IR field for the weak IR field might be the time-dependent perturbation theory treatment. It has been shown [24] that, for the IR field intensities of the order we consider, the second-order perturbation theory treatment produces results which are very close to the soft-photon approximation results. From the other hand, one can show $[22,23]$ that the soft-photon approximation agrees very well with the results of the full TDSE calculation of the photoionization process in He atom. We have reasons to believe, therefore, that Eq. (13) describes accurately the effect of the IR field on the system. This effect can be quite significant, and its proper account is necessary for the correct determination of the time delays [25].

In Fig. 4 we show results for the distribution $P_{\text {avg }}\left(v_{z}\right)$ computed using amplitudes modified by the IR field as prescribed by Eq. (13).

We can reformulate now the fitting procedure we described above as follows. If we assume that frequency of the IR field is less than the characteristic spread of the wave packet, i.e., $\Omega \ll k / \sqrt{a}$, and if we are interested in the electron energies in the vicinity of the on-shell value $k^{2} / 2$, then we can compute the right-hand side of Eq. (13) for a particular field and molecule orientation using on-shell values of the amplitude and its energy derivative. These are precisely the ingredients we used in Eq. (10) for the fitting procedure we described above. We can, therefore, reformulate the fitting procedure for the distribution $P_{\mathrm{avg}}\left(v_{z}\right)$ computed as in Eq. (12) with amplitudes modified by the IR field, using the same set of fitting parameters.

\section{CONCLUSIONS}

To summarize, we applied the time delay theory to describe evolution of the signal (the probability to record an electron at the detector) in time. We have shown that, knowing the signal as a function of time, one can devise a procedure which allows one to extract information about the differential cross sections for particular field and molecule orientations from measurements performed on randomly oriented molecules. 
To measure the resolved in time probability we suggest the use of the attosecond streaking technique allowing one to convert the temporal distribution into a velocity distribution.

The description of the ionization in the presence of the IR field relying on the soft-photon approximation gives us a means of allowing reformulation of the fitting procedure we devised for the temporal distribution into an equivalent procedure for the velocity distribution of the ionized electrons.

\section{ACKNOWLEDGMENTS}

The authors are grateful to Misha Ivanov for valuable discussions. I.A.I. and A.S.K. acknowledge support of the Australian Research Council in the form of the Discovery grant DP0985136. V.V.S. acknowledges support of the President of the Russian Federation (Grant No. MK-2344.2010.2) and the Russian Foundation for Basic Research (Grant No. 11-0100523-a). Resources of the Australian National Computational Infrastructure Facility were used.
[1] M. Schultze, M. Fiess, N. Karpowicz, J. Gagnon, M. Korbman, M. Hofstetter, S. Neppl, A. L. Cavalieri, Y. Komninos, T. Mercouris et al., Science 328, 1658 (2010).

[2] K. Klünder, J. M. Dahlström, M. Gisselbrecht, T. Fordell, M. Swoboda, D. Guénot, P. Johnsson, J. Caillat, J. Mauritsson, A. Maquet et al., Phys. Rev. Lett. 106, 143002 (2011).

[3] D. Guénot, K. Klünder, C. L. Arnold, D. Kroon, J. M. Dahlström, M. Miranda, T. Fordell, M. Gisselbrecht, P. Johnsson, J. Mauritsson et al., Phys. Rev. A 85, 053424 (2012).

[4] G. Sansone, F. Kelkensberg, J. F. Perez-Torres, F. Morales, M. F. Kling, W. Siu, O. Ghafur, P. Johnsson, M. Swoboda, E. Benedetti et al., Nature (London) 465, 763 (2010).

[5] F. Kelkensberg, W. Siu, J. F. Pérez-Torres, F. Morales, G. Gademann, A. Rouzée, P. Johnsson, M. Lucchini, F. Calegari, J. L. Sanz-Vicario et al., Phys. Rev. Lett. 107, 043002 (2011).

[6] Complete Scattering Experiments, Physics of Atoms and Molecules, edited by U. Becker and A. Crowe (Kluwer Academic Publishers, New York, 2002).

[7] S. K. Semenov and N. A. Cherepkov, J. Phys. B 36, 1409 (2003).

[8] L. Nagy, S. Borbély, and K. Póra, Phys. Lett. A 327, 481 (2004).

[9] T. Weber, A. Czasch, O. Jagutzki, A. Müller, V. Mergel, A. Kheifets, J. Feagin, E. Rotenberg, G. Meigs, M. H. Prior et al., Phys. Rev. Lett. 92, 163001 (2004).

[10] T. Weber, A. Czasch, O. Jagutzki, A. Müller, V. Mergel, A. Kheifets, E. Rothenberg, G. Meigs, M. Prior, S. Daveau et al., Nature (London) 431, 437 (2004).
[11] B. Friedrich and D. Herschbach, Phys. Rev. Lett. 74, 4623 (1995).

[12] E. P. Wigner, Phys. Rev. 98, 145 (1955).

[13] C. A. A. de Carvalho and H. M. Nussenzveig, Phys. Rep. 364, 83 (2002).

[14] A. S. Kheifets and I. A. Ivanov, Phys. Rev. Lett. 105, 233002 (2010).

[15] I. A. Ivanov, Phys. Rev. A 83, 023421 (2011).

[16] R. G. Newton, in Scattering Theory of Waves and Particles (McGraw-Hill, New York, 1966).

[17] V. V. Serov and B. B. Joulakian, Phys. Rev. A 80, 062713 (2009).

[18] J. M. Feagin, J. Phys. B 31, L729 (1998).

[19] A. Baltuška, T. Udem, M. Uiberacker, M. Hentschel, E. Goulielmakis, C. Gohle, R. Holzwarth, V. S. Yakovlev, A. Scrinzi, T. W. Hänsch et al., Nature (London) 421, 611 (2003).

[20] R. Kienberger, E. Goulielmakis, M. Uiberacker, A. Baltuska, V. Yakovlev, and F. Bammer, Nature (London) 427, 817 (2004).

[21] O. Smirnova, V. S. Yakovlev, and M. Ivanov, Phys. Rev. Lett. 94, 213001 (2005).

[22] I. A. Ivanov and A. S. Kheifets, Phys. Rev. A 83, 063411 (2011).

[23] A. Maquet and R. Taïeb, J. Mod. Opt. 54, 1847 (2007).

[24] M. Meyer, J. T. Costello, S. Düsterer, W. B. Li, and P. Radcliffe, J. Phys. B 43, 194006 (2010).

[25] S. Nagele, R. Pazourek, J. Feist, K. Doblhoff-Dier, C. Lemell, K. Tőkési, and J. Bugdörfer, J. Phys. B 44, 081001 (2011). 